\title{
Effects of Active Compression-Decompression Cardiopulmonary Resuscitation with the Inspiratory Threshold Valve in a Young Porcine Model of Cardiac Arrest
}

\author{
WOLFGANG G. VOELCKEL, KEITH G. LURIE, MIKE SWEENEY, SCOTT McKNITE, \\ TODD ZIELINSKI, PAUL LINDSTROM, COLLEEN PETERSON, VOLKER WENZEL, AND \\ KARL H. LINDNER \\ Cardiac Arrhythmia Center, Cardiovascular Division, Department of Medicine [W.G.V., K.G.L., S.M., \\ T.Z., P.L., C.P.], and Department of Anesthesiology [M.S.], University of Minnesota, Minneapolis, \\ Minnesota, U.S.A., and Department of Anesthesiology and Critical Care Medicine, Leopold-Franzens- \\ University of Innsbruck, Austria [W.G.V., V.W., K.H.L.]
}

\begin{abstract}
ABS
Active compression-decompression (ACD) cardiopulmonary
resuscitation (CPR) with the inspiratory threshold valve (ITV)
has been recently recommended by the American Heart Associ-
ation for treatment of adults in cardiac arrest (class IIb: alterna-
tive, useful intervention), but this new technique has never been
used in a pediatric population. Thus, this study was designed to
evaluate ACD + ITV CPR in a young porcine model of cardiac
arrest. After 10 min of ventricular fibrillation, and 8 min of
standard CPR, ACD + ITV CPR was performed in seven $4-$ to
6-wk-old pigs $(8-12 \mathrm{~kg})$; defibrillation was attempted 8 min
later. Within 2 min after initiation of ACD + ITV CPR, mean (
SEM) coronary perfusion pressure increased from $18 \pm 2$ to 24
\pm 3 mm Hg $(p=0.018)$. During standard versus ACD + ITV
CPR, mean left ventricular myocardial and total cerebral blood
\end{abstract}
flow was $59 \pm 21$ versus $126 \pm 32 \mathrm{~mL} \cdot \mathrm{min}^{-1} \cdot 100 \mathrm{~g}^{-1}$, and 36 \pm 7 versus $60 \pm 15 \mathrm{~mL} \cdot \mathrm{min}^{-1} \cdot 100 \mathrm{~g}^{-1}$, respectively $(p=$ $0.028)$. Six of seven animals were successfully defibrillated, and survived $>15 \mathrm{~min}$. In conclusion, the combination of ACD + ITV CPR significantly increased both coronary perfusion pressure and vital organ blood flow after prolonged standard CPR in this young porcine model of ventricular fibrillation. (Pediatr Res 51: 523-527, 2002)

Abbreviations
CPR, cardiopulmonary resuscitation
ACD, active compression-decompression
ITV, inspiratory threshold valve
ACLS, advanced cardiac life support

Pediatric cardiopulmonary arrest has a very poor prognosis, and survival rates range only from 2 to $17 \%(1,2)$. Moreover, survivors often remain neurologically disabled (1). Inasmuch as current basic and ACLS methods often fail to restore cardiac function and preserve brain function, new alternative circulation methods merit evaluation in children. In this regard, the American Heart Association and European Resuscitation Council Guidelines emphasize the need for better data regard-

Received February 2, 2001; accepted November 21, 2001.

Correspondence and reprint requests: Wolfgang Voelckel, M.D., Department of Anesthesiology and Critical Care Medicine, Leopold-Franzens-University, Anichstrasse 35, 6020 Innsbruck, Austria; e-mail: wolfgang.voelckel@uibk.ac.at

Supported by the Cardiac Arrhythmia Center, University of Minnesota, Minneapolis, MN, U.S.A.; the Department of Anesthesiology and Critical Care Medicine, LeopoldFranzens-University of Innsbruck, Austria; and the Austrian Science Foundation grant P14169-MED, Vienna, Austria.

Dr. Keith Lurie is the inventor of the Inspiratory Threshold Valve (ResQ Valve ${ }^{\mathrm{TM}}$, CPRx LLC Minneapolis, MN). As such, there is a potential conflict of interest.

Presented in part at the 30th International Educational \& Scientific Symposium, Society of Critical Care Medicine, San Francisco, CA, February 2001. ing epidemiology and treatment of pediatric cardiopulmonary arrest $(3,4)$.

Intermittent impedance to the inflow of respiratory gases during the decompression phase of CPR has recently been shown to enhance CPR efficacy (5). As such, using an ITV improved coronary perfusion pressure and myocardial blood flow in both standard (6) and ACD CPR in adult animal models (7). Furthermore, coronary perfusion pressure was significantly higher in patients receiving ACD CPR with the ITV when compared with ACD CPR alone (8). However, neither ACD CPR alone nor ACD + ITV CPR has been studied in a pediatric population yet, and a suitable ACD device for infants is not currently available. Moreover, in infants undergoing CPR, acquisition of rapid vascular access is not a simple task, even for an experienced emergency physician, and i.v. administration of vasopressor drugs may therefore be delayed, or even impossible. Whereas standard chest compressions alone are often ineffective to ensure adequate vital organ blood flow, 
the use of mechanical devices may buy time that is desperately needed to initiate ACLS strategies, especially in pediatric patients. Accordingly, this study was designed to evaluate the effects of ACD CPR with the impedance ITV on perfusion pressure, cardiocirculatory variables, and vital organ blood flow in a young porcine model. Our hypothesis was that there would be no differences in study end points between interventions.

\section{METHODS}

Surgical preparation and measurements. This project was approved by the Committee on Animal Experimentation at the University of Minnesota, and the animals were managed in accordance with the American Physiological Society, institutional guidelines, and Position of the American Heart Association on Research Animal Use, as adopted on November 11, 1984 (9). Animal care and use was performed by qualified individuals supervised by veterinarians, and all facilities and transportation comply with current legal requirements and guidelines. Anesthesia was used in all surgical interventions. All unnecessary suffering was avoided, and research was terminated if unnecessary pain or fear resulted. Our animal facilities meet the standards of the American Association for Accreditation of Laboratory Animal Care.

The study was performed according to Utstein-style guidelines (10) on seven healthy, 4- to 6-wk-old female domestic farm pigs weighing $8-12 \mathrm{~kg}$. The piglets were anesthetized with a single bolus dose of ketamine $(30 \mathrm{mg} / \mathrm{kg}$ i.m.), and pentobarbital $(15 \mathrm{mg} / \mathrm{kg}$ i.v. bolus followed by $15 \mathrm{mg} / \mathrm{kg}$ per hour i.v. infusion) given via an ear vein. Animals were intubated during spontaneous respiration with a 5-mm cuffed endotracheal tube (Mallinckrodt Critical Care, Glens Falls, NY, U.S.A.), and mechanically ventilated (model 607, Harvard Apparatus; Dover, MA, U.S.A.) at a volume-controlled setting of approximately $20 \mathrm{~mL} / \mathrm{kg}$ with room air to maintain the mean arterial $\mathrm{PCO}_{2}$ at approximately $35 \mathrm{~mm} \mathrm{Hg}$. Dextrose $5 \%$ solution and normal saline $\left(10 \mathrm{~mL} \cdot \mathrm{kg}^{-1} \cdot \mathrm{h}^{-1}\right)$ was administered continuously throughout the preparation and postresuscitation phase with an infusion pump (Flo-Gard 6201, Baxter Healthcare, Deerfield, IL, U.S.A.). Blood samples removed during the experiment were replaced by a 3 -fold higher volume of normal saline.

After intubation, the pigs were instrumented with micromanometer catheters (Millar Instruments, Houston, TX, U.S.A.) for measurement of left ventricular, ascending aortic arch, and right atrial blood pressures as previously described (7). Pressure tracings and end-tidal carbon dioxide were continuously monitored with a data acquisition and recording system [Superscope II v1. 295, GW Instruments (Somerville, MA, U.S.A.) on Power Macintosh 7100/66 computer, Apple Computer, Cupertino, CA, U.S.A., and the $\mathrm{CO}_{2} \mathrm{SMO}$ monitoring system, Novametrix Medical Systems, Wallingford, CT, U.S.A., respectively]. Coronary perfusion pressure calculated during diastole (relaxation) was defined as the arteriovenous pressure difference (time-coincident difference between aortic and right atrial pressure). Blood flow was measured with approximately $5 \times 10^{6}$ radioactively labeled microspheres $\left({ }^{141} \mathrm{Ce},{ }^{95} \mathrm{Nb}\right.$, and ${ }^{113} \mathrm{Sr}$, New England Nuclear, Dupont, Boston, MA, U.S.A.); microspheres had a mean diameter of $15 \pm$ $1.5 \mu \mathrm{m}$ and a specific activity of $10 \mathrm{mCi} / \mathrm{g}$. Each microsphere vial was placed in a water bath and subjected to ultrasonic vibration for $1 \mathrm{~min}$ before injection. Approximately $5 \times 10^{6}$ microspheres were then immediately injected into the left ventricle through the lumen of the Millar catheter. The syringe and catheter were flushed with $10 \mathrm{~mL}$ of heparinized saline. With an automatic pump (Masterflex, Barnant, Barrington, IL, U.S.A.), arterial blood was continuously withdrawn from the descending aorta at a rate of $6 \mathrm{~mL} / \mathrm{min}$ beginning just before the microspheres injection and continuing to $4 \mathrm{~min}$ after the injection. At the end of the experiment, the entire heart, cerebrum, adrenal glands, both kidneys, and small intestine were removed. The left ventricular free wall was sectioned into three layers. Aliquots of each tissue were weighed and placed into vials. Radioactivities from tissues and blood were measured with a gamma scintillation spectrometer, and blood flow values were subsequently calculated according to the method described by Heyman et al. (11).

Experimental protocol. Ventricular fibrillation was then induced with a $50-\mathrm{Hz}$ alternating current applied via two s.c. needles, and ventilation was stopped. After 10 min of untreated ventricular fibrillation, closed-chest standard CPR was performed with a pneumatically driven automatic piston device (ACD Controller, AMBU International, Glostrup, Denmark) as previously described (7); the compression rate was $100 / \mathrm{min}$ with a $50 \%$ duty cycle, and a depth of $33 \%$ of the anteroposterior diameter of the chest wall. Pressure-controlled ventilation was performed with a semiautomatic ventilator (Demand valve model L063-05R, Life Support Products Inc., Irvine, CA, U.S.A.) at a 5:1 compression-ventilation ratio at a constant flow rate $(160 \mathrm{~L} / \mathrm{min})$. After $8 \mathrm{~min}$ of CPR, the chest was then actively decompressed with a $9.0-\mathrm{cm}$ silicon suction cup (Bregler Laboratories, Offenburg, Germany) to produce a sternal displacement of $10 \%$ greater than the resting anteroposterior diameter; compression depth and frequency was maintained as described above. The compression-decompression phases were divided in half by using the point at which the suction cup passed the neutral position at the beginning of compression and the beginning of active decompression. In addition, the ITV (CPRx LLC, Minneapolis, MN, U.S.A.) was inserted into the respiratory circuit, thus occluding the airway intermittently during chest decompression without resistance to exhalation or active rescuer inhalation. As such, the one-way valve is designed to close when intrathoracic pressure is less than atmospheric pressure. In case of active inspiration, a safety check valve opens when intrathoracic pressure reaches a threshold of $-22 \mathrm{~cm} \mathrm{H}_{2} \mathrm{O}$, thus allowing air to bypass the occlusion. Vital organ blood flow during CPR was measured 4 min after starting standard CPR and 4 min after starting ACD + ITV CPR. After a total of $26 \mathrm{~min}$ of cardiac arrest, including $8 \mathrm{~min}$ of standard CPR and 8 min of ACD + ITV CPR, we attempted to restore spontaneous circulation with three direct current shocks (Lifepak 7, Physiocontrol, Redmond, WA, U.S.A.) at increasing levels of 25,50 , and $100 \mathrm{~J}$, respectively. Return of spontaneous circulation was defined as an unassisted pulse with a systolic arterial pressure $\geq 80 \mathrm{~mm} \mathrm{Hg}$, and pulse pres- 
sure of $\geq 40 \mathrm{~mm} \mathrm{Hg}$, lasting for $5 \mathrm{~min}$. All surviving animals were observed for $15 \mathrm{~min}$ after successful defibrillation. After finishing the experimental protocol, the animals were killed and underwent necropsy to check for correct positioning of the catheters and damage to the rib cage and internal organs and to harvest the internal organs.

Statistical analysis. Values are expressed as mean \pm SEM. Comparisons of hemodynamic measurements within groups were performed with the paired $t$ test. Because blood flow data were distributed unevenly, the Wilcoxon signed rank test was used. Statistical significance was considered to be at $p<0.05$ after Bonferroni correction for multiple comparisons.

\section{RESULTS}

During standard CPR, coronary perfusion pressure and mean arterial pressure progressively declined from $25 \pm 3$ to $18 \pm 2$ $\mathrm{mm} \mathrm{Hg}$, and $43 \pm 4$ to $35 \pm 1 \mathrm{~mm} \mathrm{Hg}$, respectively (NS). The decrease in perfusion pressures was further associated with a decline in end-tidal carbon dioxide levels (NS; Fig. 1). When standard CPR was replaced with active ACD + ITV CPR, left ventricle, global cerebral, and renal blood flow each increased significantly $(p<0.03)$. Moreover, brain blood flow during ACD + ITV CPR was even comparable with prearrest values $(p=0.5$; Table 1$)$. Furthermore, oxygen extraction ratio decreased during ACD + ITV CPR. As such, we found arterial and mixed venous $\mathrm{PO}_{2}$ during ACD + ITV CPR to be comparable with prearrest values. By contrast, despite higher arterial $\mathrm{PO}_{2}$ during standard $\mathrm{CPR}$, mixed venous values were significantly lower during this phase of the protocol when compared with baseline values. In this regard, it is noteworthy that ventilation was performed with pure oxygen during CPR. When initiating ACD + ITV CPR, arterial and, subsequently, mixed venous $\mathrm{PCO}_{2}$ significantly increased, although controlled minute ventilation remained unchanged throughout the study (Table 2). Mean arterial pressure and end-tidal carbon dioxide values during the next $8 \mathrm{~min}$ of ACD + ITV CPR were significantly higher when compared with the last value after 8 min of standard CPR, despite the prolonged duration of cardiac arrest. The increase in coronary perfusion pressure during ACD + ITV CPR was also obvious and statistically significant $(p=0.018$ and $p=0.037$ for values at minute 10 and 12 versus minute 8 during standard CPR; Fig. 1). All but one animal were successfully defibrillated, and survived for $15 \mathrm{~min}$ without any pharmacologic intervention; the mean number of shocks needed for defibrillation was $2.4 \pm 0.3$. Necropsy confirmed appropriate catheter positions, and revealed no injuries of the rib cage or intrathoracic organs in any animals caused by the suction cup used.

\section{DISCUSSION}

Results from the current study using a young porcine model of cardiac arrest demonstrate that the combination of ACD + ITV CPR significantly improved perfusion pressures and, subsequently, vital organ blood flow above a threshold that rendered successful defibrillation likely. Accordingly, in six of seven animals, spontaneous circulation was restored after prolonged CPR of 26 min of cardiac arrest without any pharmacologic intervention. It is actually remarkable that our animals had an increase in perfusion pressures and vital organ blood flow when global ischemia usually reaches a point that renders many CPR interventions barely effective. Moreover, the fact that six of seven animals had return of spontaneous circulation after a total of 26 min of low or no flow suggests that by using the ITV, we bought some time to increase the window for successful resuscitation.

In many children undergoing CPR, outcome is dismal when ACLS cannot be provided instantly. Moreover, administration of vasopressor drugs may play an import role to restore vital organ blood flow, but gaining an i.v. infusion catheter is a major challenge in the treatment of these patients. Accordingly, new devices designed to improve vital organ blood flow during CPR are needed to buy time, which is desperately needed to render even delayed ACLS therapies effective. Recently, ACD CPR has been shown to improve survival after cardiac arrest (12). When inflow of respiratory gases is limited with the ITV selectively during the decompression phase of CPR, venous return into the chest is further enhanced, thus improving ACD CPR efficacy $(5,7)$. In adult patients in cardiac arrest, the combination of ACD CPR and the ITV resulted in near normal blood pressures $(110 / 55 \mathrm{~mm} \mathrm{Hg})$ during prolonged cardiac arrest (8). This approach has not been evaluated in the treatment of pediatric patients. The current results describe the first evaluation of ACD + ITV CPR in a young porcine model of cardiac arrest. Although performed with an automated device, we found that ACD CPR is a technique tha can be easily adapted to the pediatric setting. The concept of ACD CPR is further supported by the fact that the juvenile chest wall lacks an intrinsic tendency to rebound when released from compression during CPR. As such, Dean et al. (13) demonstrated that inadequate chest recoil, development of static chest deforma-

Table 1. Vital organ blood flow before cardiac arrest, during standard CPR and during ACD CPR with the ITV

\begin{tabular}{lccc}
\hline \multicolumn{1}{c}{ Variable } & Prearrest & STD CPR & ACD + ITV CPR \\
\hline Left ventricle $\left(\mathrm{mL} \cdot \mathrm{min}^{-1} \cdot 100 \mathrm{~g}^{-1}\right)$ & $318 \pm 53$ & $59 \pm 21$ & $126 \pm 32^{*}$ \\
Endo/epi ratio & $1.18 \pm 0.06$ & $1.08 \pm 0.22$ & $1.20 \pm 0.163$ \\
Brain $\left(\mathrm{mL} \cdot \mathrm{min}^{-1} \cdot 100 \mathrm{~g}^{-1}\right)$ & $54 \pm 5$ & $36 \pm 7$ & $60 \pm 15^{*}$ \\
Right $\mathrm{kidney}\left(\mathrm{mL} \cdot \mathrm{min}^{-1} \cdot 100 \mathrm{~g}^{-1}\right)$ & $321 \pm 61$ & $12 \pm 7$ & $40 \pm 11 \dagger$ \\
Left $\mathrm{kidney}\left(\mathrm{mL} \cdot \mathrm{min}^{-1} \cdot 100 \mathrm{~g}^{-1}\right)$ & $340 \pm 65$ & $99 \pm 2$ & $40 \pm 11 \dagger$ \\
Adrenal glands $\left(\mathrm{mL} \cdot \mathrm{min}^{-1} \cdot 100 \mathrm{~g}^{-1}\right)$ & $332 \pm 65$ & $24 \pm 8$ & $155 \pm 45$ \\
Small intestine $\left(\mathrm{mL} \cdot \mathrm{min}^{-1} \cdot 100 \mathrm{~g}^{-1}\right)$ & $38 \pm 9$ & $27 \pm 7$ \\
\hline
\end{tabular}

All variables are given as mean \pm SEM. Prearrest indicates measurements before induction of ventricular fibrillation.

Abbreviations used: STD, standard; Endo/epi, endocardial/epicardial blood flow ratio.

$* p=0.028 v s$ standard CPR; $\dagger p=0.018 v s$ standard CPR. 
Table 2. Hemodynamic and blood gas variables before cardiac arrest, during standard CPR, during ACD CPR with the ITV, and during the postresuscitation phase in piglets

\begin{tabular}{|c|c|c|c|c|c|}
\hline \multirow[b]{2}{*}{ Variable } & \multirow[b]{2}{*}{ Prearrest } & \multirow{2}{*}{$\begin{array}{l}\text { STD CPR, } \\
4 \mathrm{~min}\end{array}$} & \multirow{2}{*}{$\begin{array}{l}\mathrm{ACD}+\mathrm{ITV} \\
\mathrm{CPR}, 12 \mathrm{~min}\end{array}$} & \multicolumn{2}{|c|}{ Postresuscitation phase (min)* } \\
\hline & & & & 5 & 15 \\
\hline Heart rate $(\mathrm{bpm})$ & $165 \pm 10$ & - & - & $155 \pm 5$ & $185 \pm 20$ \\
\hline Arterial $\mathrm{pH}$ (units) & $7.41 \pm .01$ & $7.19 \pm .04$ & $7.03 \pm .02 \ddagger$ & $7.06 \pm .04$ & $7.10 \pm .04$ \\
\hline Arterial $\mathrm{Po}_{2}(\mathrm{~mm} \mathrm{Hg})$ & $78 \pm 5$ & $155 \pm 40$ & $115 \pm 20$ & $70 \pm 5$ & $80 \pm 5$ \\
\hline Arterial $\mathrm{PCO}_{2}(\mathrm{~mm} \mathrm{Hg})$ & $37 \pm 2$ & $49 \pm 6$ & $69 \pm 5 \ddagger$ & $66 \pm 6$ & $60 \pm 6$ \\
\hline
\end{tabular}

All variables are given as mean \pm SEM. Prearrest indicates measurements before induction of cardiac arrest.

Abbreviation used: STD, standard.

$* n=6 ; \dagger p<0.01 \mathrm{ACD}+\mathrm{ITV} v$ STD CPR; $\$ p<0.05$ ACD + ITV vs STD CPR; no statistical comparisons were performed with prearrest and postresuscitation values.

tion, and limitation of pulsatile chest wall movement occurred in 8-wk-old pigs when relaxation time was as short as $250-300$ ms during CPR. Because venous blood return to the chest and diastolic right atrial pressure are highly dependent on the intrathoracic pressure drop during the relaxation phase of CPR, we suggest that ACD + ITV CPR in pediatric patients may even contribute more than in adults to the concept of enhancing the bellowslike action of the chest. Moreover, the more compliant juvenile chest is likely to facilitate manually performed ACD CPR with a hand-held device consisting of a suction cup and handle.

When combining both ACD CPR and the ITV, left ventricular blood flow was more than doubled, and cerebral blood flow even exceeded baseline values in our study. Both effects are most likely correlated to the increase in mean arterial pressure during ACD + ITV CPR, although cerebral blood flow may be influenced by the degree of arterial hypercarbia. Although mixed venous, arterial, and end-tidal $\mathrm{PCO}_{2}$, which may serve as prognosticators of cardiac resuscitability (1416), significantly increased during ongoing ACD + ITV CPR, arterial and mixed venous $\mathrm{pH}$ values further decreased. In this regard, we speculate that at least three mechanisms may contribute to this observed effect of ACD + ITV CPR: first, intermittent airway occlusion is likely to cause a decrease in minute ventilation despite the fact that pressure-controlled ventilation was maintained at a constant flow rate. It has been recognized that intermittent airway occlusion during prolonged ACD CPR may result in some alveolar collapse, with a decrease in calculated static lung compliance and subsequently in impaired lung function (17). As such, we observed lower arterial $\mathrm{PO}_{2}$ and higher $\mathrm{PCO}_{2}$ values 4 min after initiating ACD + ITV CPR. Although pulmonary edema did not occur in any of the piglets, strategies to improve oxygenation during ACD + ITV CPR may be needed to minimize the aforementioned side effects. In this regard, we recently demonstrated that the use of positive end-expiratory pressure may reduce alveolar collapse during ACD + ITV CPR and, even more, is likely to result in an increase in indirect myocardial compression (17). Second, prolonged cardiac arrest and systemic hypoperfusion will result in low arterial and mixed venous $\mathrm{pH}$ values because of global hypoxia and hypercarbic acidosis. Finally, we speculate that the observed decrease of arterial and mixed venous
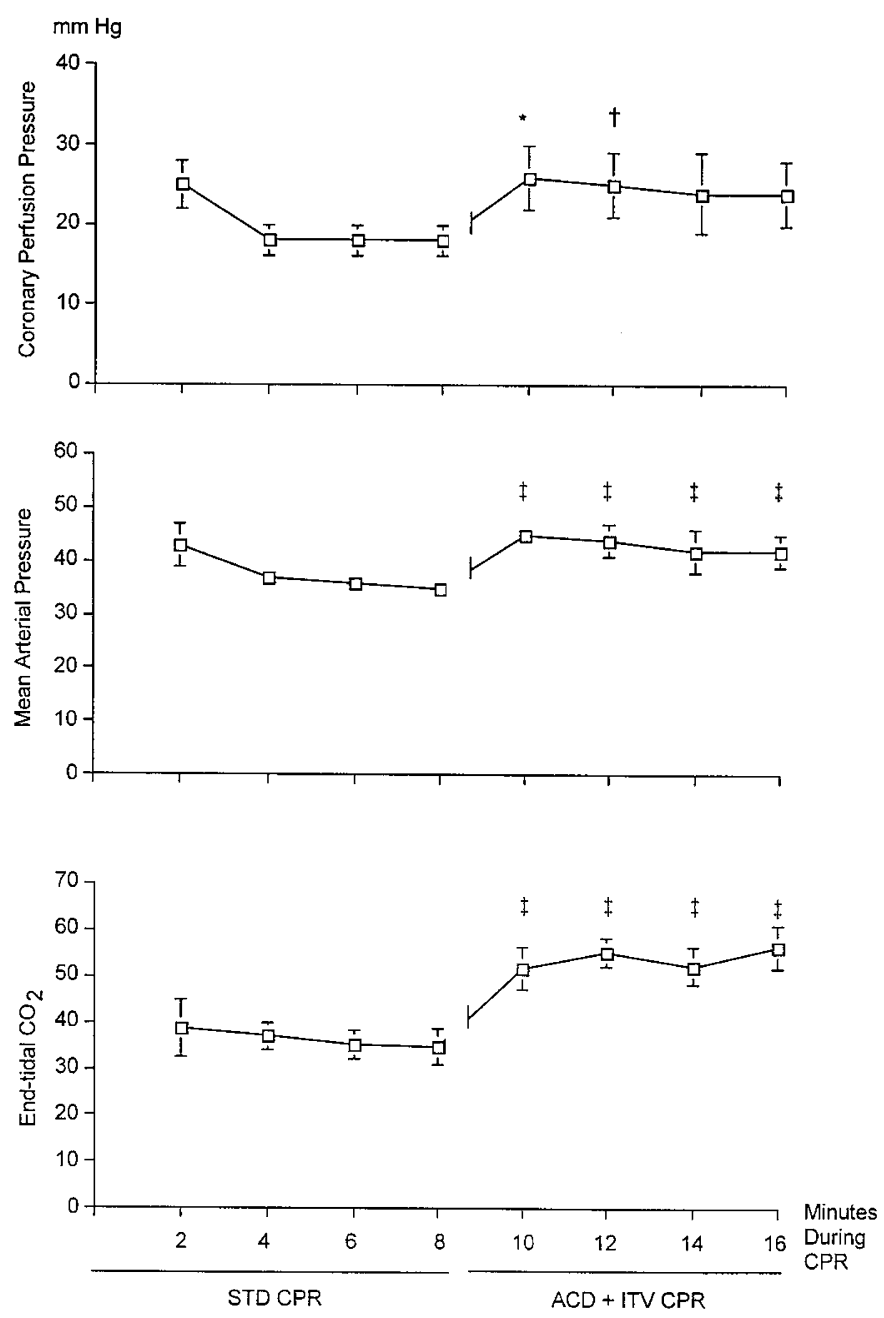

Figure 1. Mean coronary perfusion pressure, mean arterial pressure, and end-tidal $\mathrm{PCO}_{2}$ during standard (STD) and ACD CPR with the ITV. * $p=0.018$; $\dagger p=0.037 ; \ddagger p<0.001 v s$ last value during STD CPR at minute 8 . Time during $\mathrm{CPR}$ is given in minutes.

$\mathrm{pH}$ values as well as the increase of arterial and mixed venous $\mathrm{PCO}_{2}$ may indicate effective circulatory assistance, and reflects systemic blood flow and reperfusion washout (18). As such, we found no paradox between the $\mathrm{pH}$ and $\mathrm{PCO}_{2}$ in arterial and mixed venous blood $(16,18,19)$. Further supporting this hypothesis of improved systemic circulation were findings of 
lower oxygen extraction ratio during ACD + ITV CPR when compared with standard CPR alone. In this regard it is noteworthy that six of seven animals had return of spontaneous circulation without pharmacologic intervention. When compared with an adult animal study consisting of 4 min of cardiac arrest and $18 \mathrm{~min}$ of CPR, neither standard CPR alone nor treatment with epinephrine rendered defibrillation successful (20). Accordingly, results from our present and a previous study (17) may provide further evidence that ACD + ITV CPR may be considered as an effective tool to gain time during prolonged CPR efforts. Nevertheless, ongoing low-flow states during prolonged cardiac arrest will result in profound tissue acidosis and depletion of endogenous catecholamine stores. Both mechanisms are likely to limit the potential of solely mechanical CPR without any pharmacologic support.

Although we are aware that asphyxiation and shock are the most likely causes of cardiac arrest in infants, we chose an established porcine model of ventricular fibrillation and prolonged nonintervention cardiac arrest interval for this initial evaluation of ACD + ITV CPR in a porcine model. Accordingly, some limitations of the present study should be noted. First, different cardiac arrest scenarios such as hypoxic or hypovolemic cardiac arrest may interfere with the efficacy of ACD + ITV CPR and significantly affect outcome. Second, we are not able to report on the effects of ACD CPR alone, and further animal studies are needed to allow a more comprehensive estimation of the efficiency of each respective CPR technique. Finally, data from an animal model may not be transferred into a clinical setting. However, inasmuch as ACD CPR has been performed with success in adult patients, our data suggest that this technique could be feasible in pediatric patients and may merit clinical evaluation if the observed hemodynamic effects can be reproduced in further animal studies.

In conclusion, the combination of ACD + ITV CPR both significantly increased coronary perfusion and preserved vital organ blood flow after prolonged standard CPR in this young porcine model of ventricular fibrillation.

\section{REFERENCES}

1. Schindler MB, Bohn D, Cox PN, McCrindle BW, Jarvis A, Edmonds J, Barker G 1996 Outcome of out-of-hospital cardiac or respiratory arrest in children. N Engl J Med 336:1473-1479
2. Zaritsky AL 1998 Recent advances in pediatric cardiopulmonary resuscitation and advanced life support. New Horiz 6:201-211

3. [No author] 2000 Guidelines 2000 for cardiopulmonary resuscitation and emergency cardiovascular care. Circulation 102(suppl):I1-I384

4. [No author] 2000 Guidelines 2000 for cardiopulmonary resuscitation and emergency cardiovascular care: International Consensus on Science. Resuscitation 46:1-447

5. Lurie K, Voelckel W, Plaisance P, Zielinski T, McKnite S, Kor D, Sugiyama A, Sukhum P 2000 Use of an inspiratory impedance threshold valve during cardiopulmonary resuscitation: a progress report. Resuscitation 44:219-230

6. Lurie KG, Mulligan KA, McKnite S, Detloff B, Lindstrom P, Lindner KH 1998 Optimizing standard cardiopulmonary resuscitation with an inspiratory impedance threshold valve. Chest 113:1084-1090

7. Lurie KG, Coffen P, Shultz J, McKnite S, Detloff B, Mulligan K 1995 Improving active compression-decompression cardiopulmonary resuscitation with an inspiratory impedance valve. Circulation 91:1629-1632

8. Plaisance P, Lurie KG, Payen D 2000 Inspiratory impedance during active compression-decompression cardiopulmonary resuscitation: a randomized evaluation in patients in cardiac arrest. Circulation 101:989-994

9. National Institutes of Health 1985 Guide for the Care and Use of Laboratory Animals. U.S. Government Printing Office, Washington, D.C., publication 86-23

10. Idris AH, Becker LB, Ornato JP, Hedges JR, Bircher NG, Chandra NC, Cummins RO, Dick W, Ebmeyer U, Halperin HR, Hazinski MF, Kerber RE, Kern KB, Safar P, Steen PA, Swindle MM, Tsitlik JE, von Planta I, von Planta M, Wears RL, Weil MH 1996 Utstein-style guidelines for uniform reporting of laboratory CPR research. A statement for healthcare professionals from a Task Force of the American Heart Association, the American College of Emergency Physicians, the American College of Cardiology, the European Resuscitation Council, the Heart and Stroke Foundation of Canada, the Institute of Critical Care Medicine, the Safar Center for Resuscitation Research, and the Society for Academic Emergency Medicine. Resuscitation 33:69-84

11. Heymann MA, Payne BD, Hoffmann JI, Rudolph AM 1977 Blood flow measurements with radionuclide-labeled particles. Progr Cardiovasc Dis 20:55-79

12. Plaisance P, Lurie KG, Vicaut E, Adnet F, Petit JL, Epain D, Ecollan P, Gruat R, Cavagna P, Biens J, Payen D 1999 A comparison of standard cardiopulmonary resuscitation and active compression-decompression resuscitation for out-of-hospital cardiac arrest. French Active Compression-Decompression Cardiopulmonary Resuscitation Study Group. N Engl J Med 341:569-575

13. Dean JM, Koehler RC, Schleien CL, Berkowitz I, Michael JR, Atchison D, Rogers MC, Traystman RJ 1990 Age-related effects of compression rate and duration in cardiopulmonary resuscitation. J Appl Physiol 68:554-560

14. Berg RA, Henry C, Otto CW, Sanders AB, Kern KB, Hilwig RW, Ewy GA 1996 Initial end-tidal $\mathrm{CO}_{2}$ is markedly elevated during cardiopulmonary resuscitation after asphyxial cardiac arrest. Pediatr Emerg Care 12:245-248

15. Callaham M, Barton C 1990 Prediction of cardiopulmonary resuscitation from end-tidal carbon dioxide concentration. Crit Care Med 18:358-362

16. Gazmuri RJ, von Planta M, Weil MH, Rackow EC 1989 Arterial $\mathrm{PCO}_{2}$ as an indicator of systemic perfusion during cardiopulmonary resuscitation. Crit Care Med 17:237240

17. Voelckel WG, Lurie KG, Zielinski T, McKnite S, Plaisance P, Wenzel V, Lindner KH 2001 The effects of positive end-expiratory pressure during active compression decompression cardiopulmonary resuscitation with the inspiratory threshold valve. Anesth Analg 92:967-974

18. Angelos MG, DeBehnke DJ, Leasure JE 1992 Arterial blood gases during cardiac arrest: markers of blood flow in a canine model. Resuscitation 23:101-111

19. Grundler W, Weil MH, Rackow EC 1986 Arteriovenous carbon dioxide and $\mathrm{pH}$ gradients during cardiac arrest. Circulation 74:1071-1074

20. Wenzel V, Lindner KH, Krismer AC, Voelckel WG, Schocke MF, Hund W, Witkiewicz M, Miller EA, Klima G, Wissel J, Lingnau W, Aichner FT 2000 Survival with full neurological recovery, and no cerebral pathology after prolonged cardiopulmonary resuscitation with vasopressin in pigs. J Am Coll Cardiol 35:527-533 